\title{
Synthetic Aperture Radars for Humanitarian Purposes: Products and Opportunities
}

\author{
Donato Amitrano, Gerardo Di Martino, Antonio \\ Iodice, Daniele Riccio, Giuseppe Ruello \\ Department of Electrical and Information Technology \\ Engineering \\ University of Napoli Federico II \\ Napoli, Italy
}

\author{
Maria Nicolina Papa, Fabio Ciervo \\ University of Salerno \\ Salerno, Italy \\ mnpapa@unisa.it \\ Youssouf Koussoube \\ Departement de Geologie, UFR/SVT \\ University of Ouagadougou \\ Ouagadougou, Burkina Faso
}

\begin{abstract}
In this paper we present the available products and the future opportunities derived by the use of Synthetic Aperture Radar data for humanitarian projects. A set of images relevant to an area of the Sahel, in Burkina Faso have been used for supporting the proposed claims. Along with the technical problems related with the image interpretation, we focus on the economic accessibility of the data, that mainly limited the use of SAR images in low income countries. The future availability of free access data makes their use very attractive, provided that appropriate knowledge is available for interpreting the images.
\end{abstract}

Index Terms- Synthetic Aperture Radar; agricolture; value added products.

\section{INTRODUCTION}

Remote sensing instruments provide a huge amount of information on the Earth's surface. Products acquired by remote passive and active sensors provide information at several scales and with several revisit time, whose use could be of great impact for the solution of land related problems in humanitarian projects [1] - [3].

Nevertheless, remote sensing is still scarcely used in humanitarian applications, due to the high cost of images and the difficulties related with the processing and the interpretation of the data.

Therefore, one of the major challenges for the remote sensing community is the development of new and more user friendly processing instruments and innovative products which can be easily interpreted and therefore used in humanitarian project.

Purpose of this paper is to present the potentialities of Synthetic Aperture Radar (SAR) sensors, supported by the results of a project developed in Burkina Faso with data acquired by the Italian sensor Cosmo-Skymed (CSK) [4]-[6]. CSK provided $3 \mathrm{~m}$ resolution images whose interpretation can be arduous for non-expert users.

In Section 2, the rationale of the proposed approach is presented along with a series of potential applications and results from the above cited project. In particular, we present a new approach for representing in simple form information about features of interest of the observed area: land cover maps, digital elevation models, vegetation growth monitoring and surface water index are retrieved by properly combining amplitude and phase SAR data. The approach is tailored on semi-arid regions. The alternation of a wet and a dry season is exploited for developing the proposed procedure.

In Section 3, we discuss how the retrieved information may be used in humanitarian project. In addition, we discuss the feasibility of the use of satellite data also in low-income countries, thanks to the new policies of the European Space Agency of providing the data for free and with open access [7], [8]. Such an approach opens the way to a wide range of possible applications. In the near future, the economic problems related with the data acquisition will be reduced and the only requirements for exploiting the data will be the capacity of reading, processing and interpreting the available data. A framework for the operational use of SAR data from the space agencies to the end users is also presented. Section 4 is dedicated to the conclusive remarks.

\section{INNOVATIVE PRODUCTS}

Synthetic Aperture radars are active sensors that measure the amount of energy backscattered by the observed surfaces. SAR images represent an estimation of the reflectivity function of the scene (see, as instance, the intensity maps depicted in Fig. 1), whose non-intuitive interpretation sometimes discourage the potential users. However, the actual perspective of the creation of free access databases, as already happens for Landsat data, makes the use of SAR images very attractive in many practical applications.

The role of multi-disciplinary research is crucial for the development of user friendly algorithms and products of immediate interpretation for the users. Moving by this challenge, one of the aims of this work is to highlight the potentiality and the usefulness of the SAR Level 2 products. Generally speaking, a Level 2 product is a product useroriented, able to provide geophysical information without any further processing. 


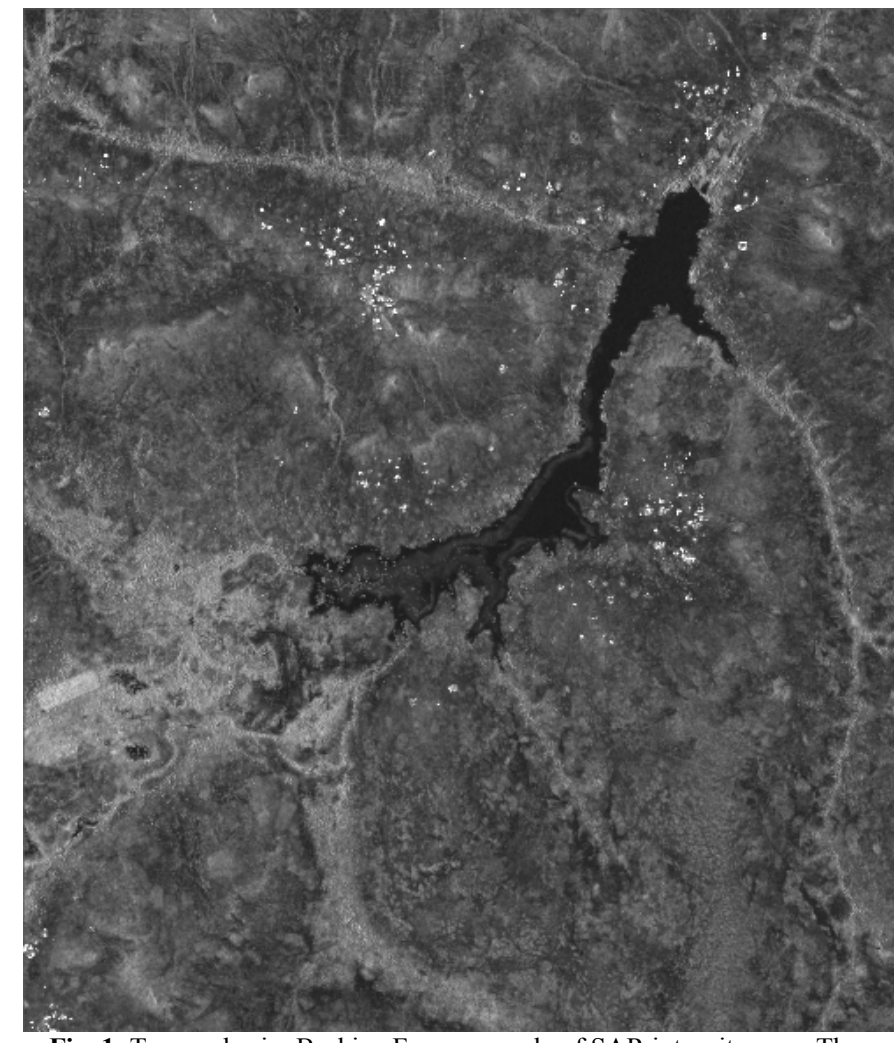

Fig. 1: Tougou basin, Burkina Faso: example of SAR intensity map. The imaged area is about $18 \mathrm{Km} \times 15 \mathrm{Km}$.

In the following, we present the physical principles for some of the most significant methods for retrieving information from SAR data. The principles are presented along with the results coming from the processing of a set of 12 Stripmap CSK images, relative to the Yatenga region, located in the North of Burkina Faso. In particular, the region of interest includes the basin of Tougou, which is located about $20 \mathrm{~km}$ easting with respect to Ouahigouya, the capital of the district. The studied area (whose reference coordinates are: $13^{\circ} 40^{\prime} 50^{\prime \prime} \mathrm{N}, 2^{\circ} 12^{\prime} 50^{\prime} \mathrm{W}$ ) is characterized by a three months wet season (from June to September) and a 9 months dry season (from October to May).

The time series contains images from 2010, June 12 to 2011, December 12. In Fig. 1, an example of SAR image representing the basin of Tougou is shown. The Tougou dam is in the upper-right corner of the image. The image is represented in the geometry of acquisition of the sensor: azimuth (up-down direction) / slant range(left-right direction).

Data gathered by SAR representing land cover, digital elevation model, crop estimation and eroded areas are presented in the next subsections.

\section{A. Land Cover}

The availability of multi-time series allows to combine different images in order to retrieve information on the observed areas.

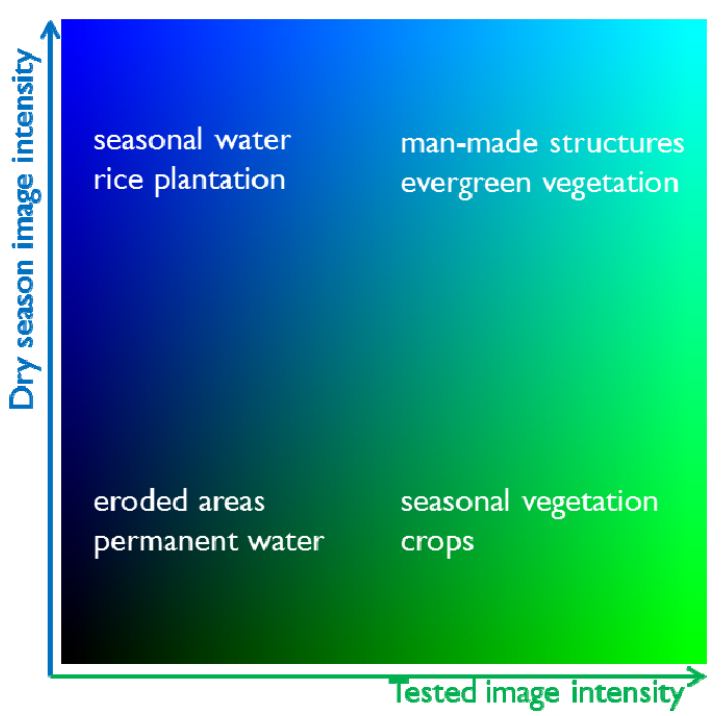

Fig. 2: Interpretative tool for Green-Blue images.

By comparing the amplitudes of different images, it is possible to obtain false color images, able to emphasize the land cover of a given area, provided that a model for their interpretation is provided. In semi-arid regions, at the end of the dry season, it is reasonable to expect that the land will be deprived of both surface water and vegetation. In terms of the electromagnetic return, this will provoke a minimum amount of reflection, and therefore, weak signal will be measured by the SAR. With the starting of the rainy season, the terrains will absorb water, the reservoirs will accumulate surface water and the corresponding electromagnetic backscattering will be deeply modified. Therefore, it is possible to classify the observed area in a given date as a function of the amplitude variation with respect to a reference dry season image.

Let us load the dry season reference image on the blue band and the test image on the green band. Therefore, it is possible to define the interpretation table presented in Fig. 2 of the corresponding color composite image for classifying pixels belonging to different land uses.

A third information can be introduced, by loading on the red band the interferometric coherence, so that the urban settlements can be identified as high amplitude and high coherence scatterers. In Fig. 3 we present an example of a land cover map, created on the basis of the rationale introduced above. The green areas represent the presence of seasonal vegetation; the blue band is related to the seasonal water. Dark areas are indicators of eroded areas.

\section{B. Digital Elevation Model}

Couples of SAR images can be used for obtaining topographic information on the areas of interest in a cheapest and quickest way with respect to traditional techniques. 

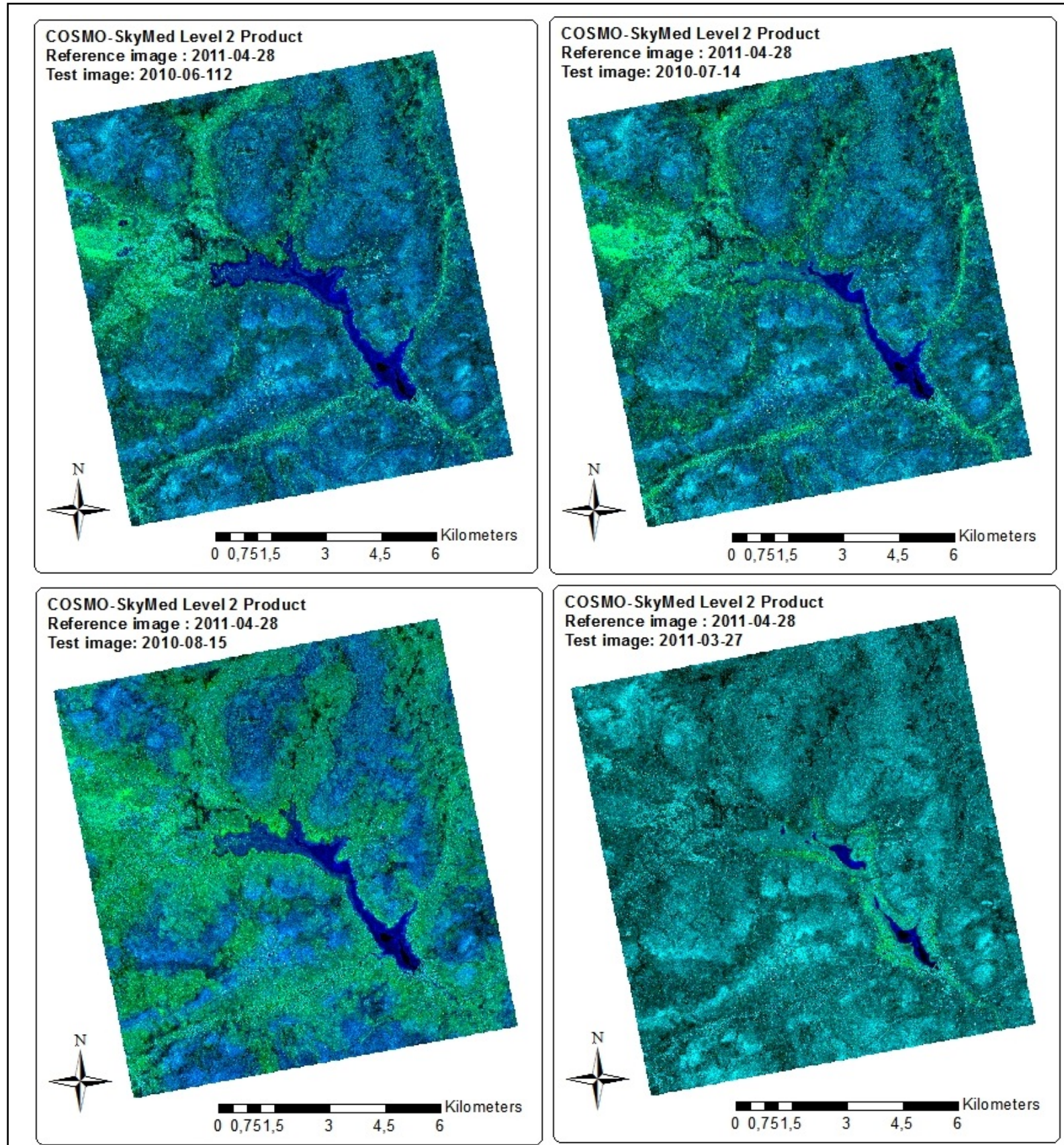

Fig. 3: Tougou basin, Burkina Faso: land cover maps. Reference image (blue band): 2011/04/28 (dry season). Test images (green band) from left to right and up to down: 2010/06/12, 2010/07/14, 2010/08/15, 2011/03/27.

In order to show the potentiality of such an approach, we present the result of the processing of a pair of CSK images acquired at the end of April, 2011, with a temporal separation of 1 day. Such a couple allowed the extraction of a digital elevation model (DEM) that was used to extract the topographic information. Thanks to the limited temporal shift and to the absence of significant vegetation at the end of the dry season, the interferometric coherence (i.e., the DEM quality) is very high in most of the observed area. The development of a complete interferometric chain allowed the production of the georeferenced DEM presented in Fig. 4.
Details on the DEM accuracy and have been already presented in [5]. In low-income countries, the availability of topographical information can be rare, expensive or scarcely accurate. The obtained product provided a better resolution if compared with any other available product in the area. 


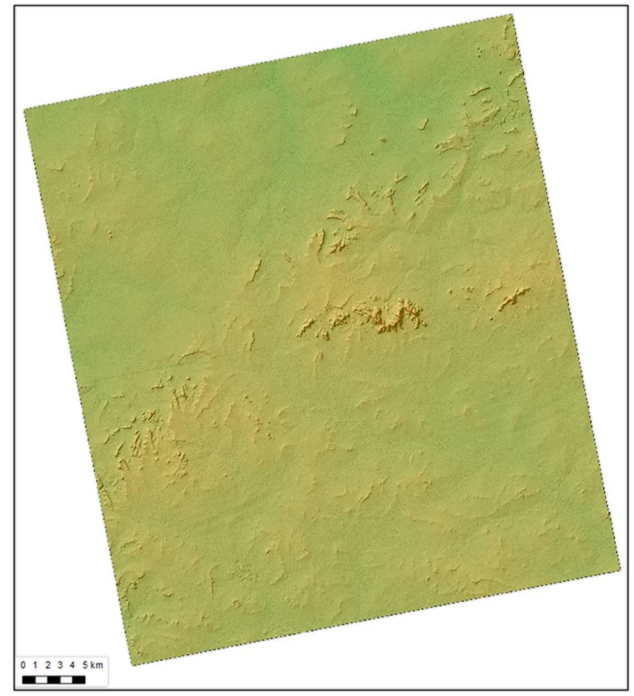

InSAR Digital Elevation Model

Fig. 4: Digital Elevation model of the area of Ouahigouya

\section{Crop monitoring}

One of the most attractive aspects related with the availability of multi-temporal images is the chance of creating maps of indexes which can be, in turn, transformed in classification maps. The vegetation growth can be monitored by exploiting the backscattering enhancement in SAR images due to the volume contribution of the plant foliage. In the dry season the expected return from the bare soil is very weak, and during the wet season, the backscattering increases as a function of the amount of vegetation in the field. A vegetation index can be therefore derived by the comparison of the amplitude returns of the acquisitions taken in the dry and the wet season. In Figure 5 we present the evolution of a vegetation index, defined as a normalized difference between the test image and a reference image relative to the dry season, in four different dates (June, July, August and March). Such a map allows the quantification of the area covered by vegetation during the year.
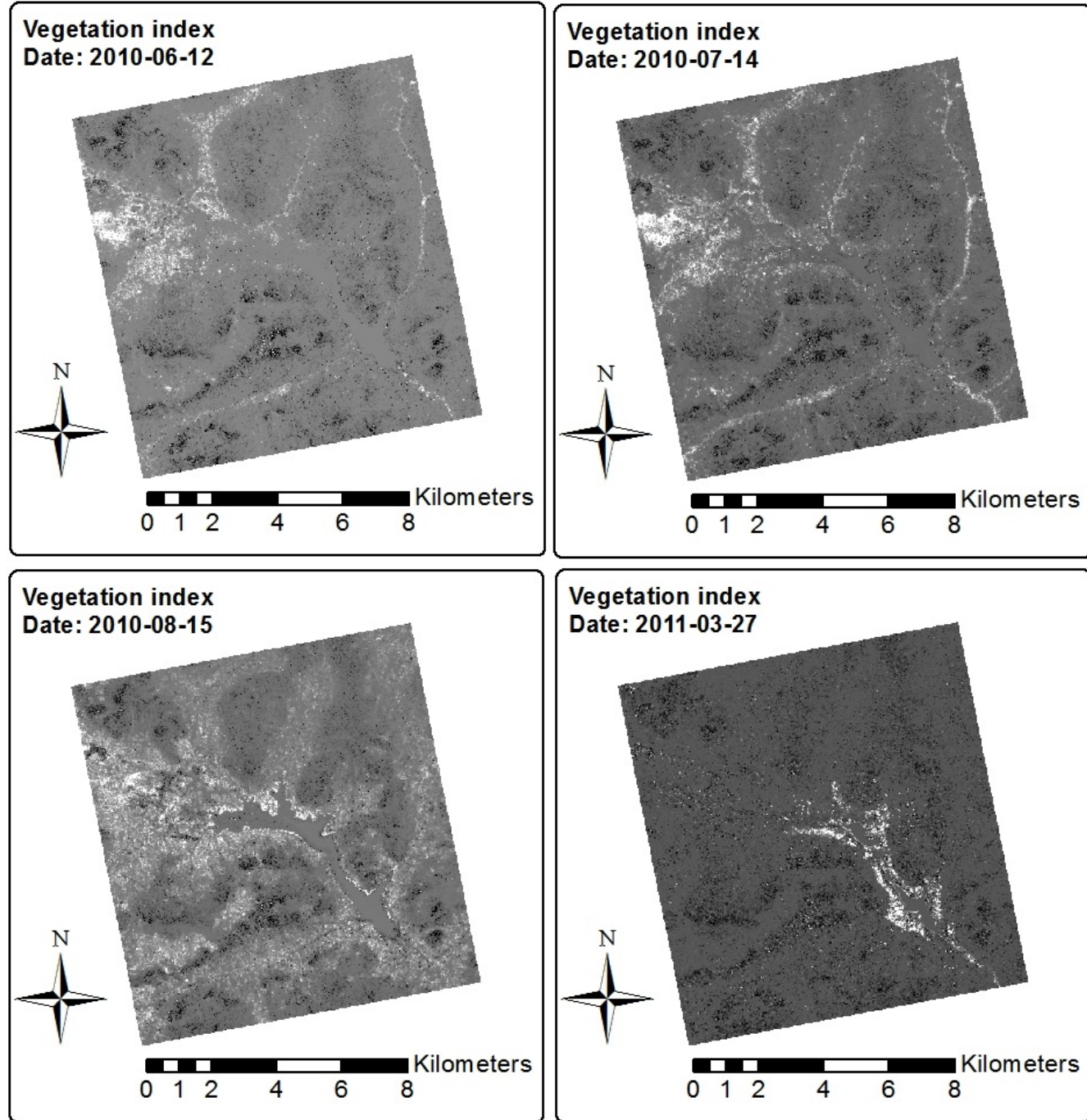

Fig. 5: Tougou basin, Burkina Faso: vegetation index maps. Reference image: 2011/04/28 (dry season). Test images from left to right and up to down: 2010/06/12, 2010/07/14, 2010/08/15, 2011/03/27. Index values range from 0 (black) to 1 (white). 

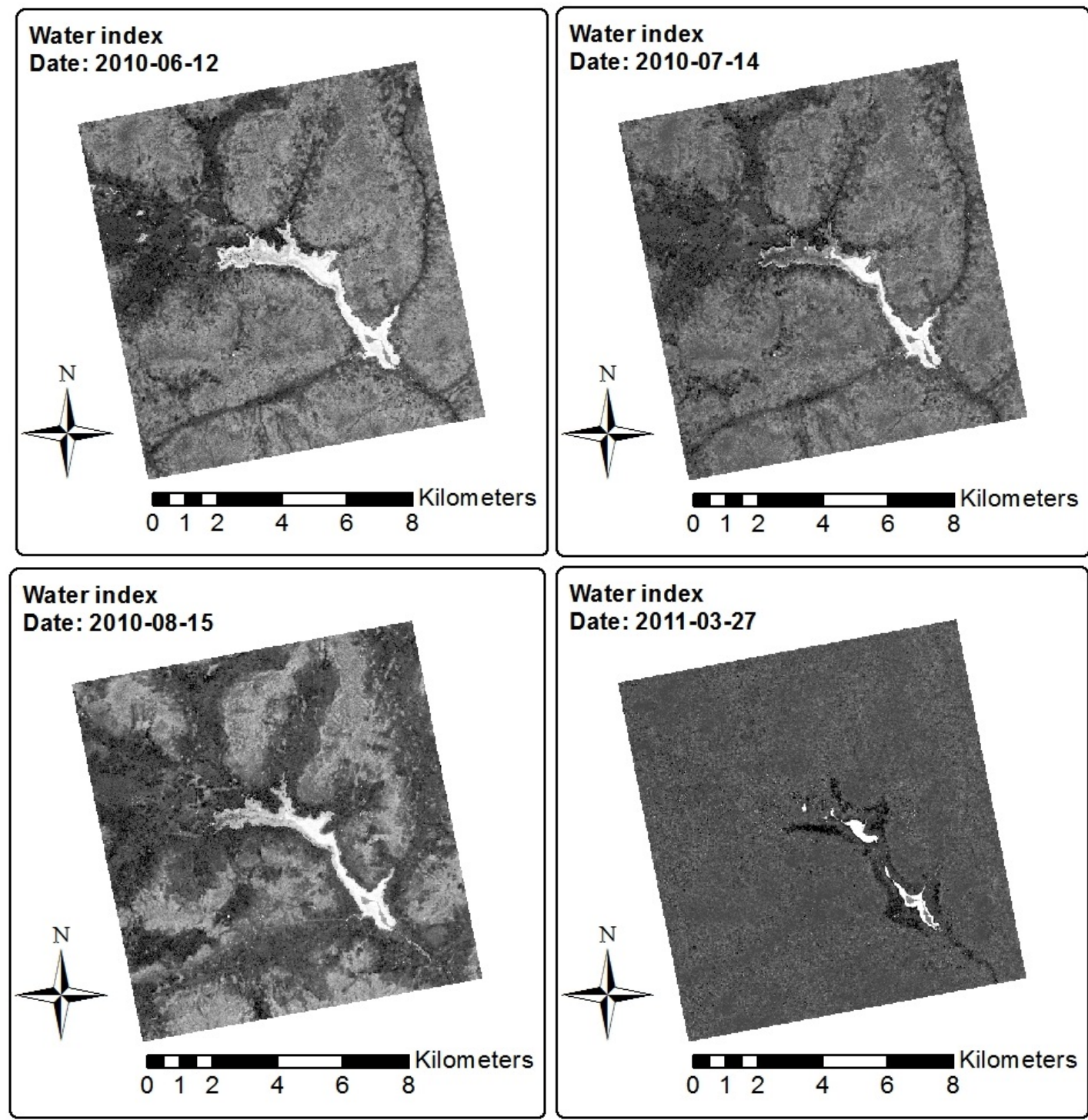

Fig. 6: Tougou basin, Burkina Faso: water index maps. Reference image: 2011/04/28 (dry season). Test images from left to right and up to down: 2010/06/12, 2010/07/14, 2010/08/15, 2011/03/27. Index values range from 0 (black) to 1 (white).

\section{Surface water monitoring}

Also the surface water cicle is, of course, related to the precipitation input and the high evaporation rate of the area. In the dry season, when the water intakes are empty, the signal backscattered by the terrain (i.e. the SAR image amplitude) is higher with respect to the signal backscattered during the rainy season, relevant to areas covered by surface water. By using a similar approach to the case presented in the previous paragraph, we can define a surface water index, as normalized difference between the dry season and the test wet season. The obtained maps are presented in Fig. 6. It is straightforward to note that from June to August the water surface increases thanks to the abundant rainfalls. The analysis of the maps during the dry season reveals the possibility of monitoring the amount of available water.

\section{FUTURE OPPORTUNITIES}

The limited but significant set of examples presented in the previous section shows that the availability of high resolution SAR data provide a huge amount of information of great interest for humanitarian projects.

First examples are the early estimation of the seasonal crop, based on the vegetation index and of the best location for counter-season agriculture, based on the water index.

Another application concerns the drawing of erosion maps [4], [9]. To this purpose, the most widely used method is USLE [10] that is based on the superposition of information obtained by heterogeneous sources, including the topographic information (DEM) and land cover [9]-[10].

Climate change monitoring can be also achieved through the exploitation of the proposed maps. In fact, variation of the expected trends of vegetation cover and soil water retention can be indicators of climate changes. 
Hydrological models useful for the improvement of water resource management can be supported by the information presented in Section 2.

The scarce use of remote sensing products in humanitarian projects is due not only to technical, but also to economic issues. The high cost of SAR images limited so far their use. Anyway, the next missions of the European Space Agency are planned to provide free and open access data, opening the way to a huge amount of possible applications.

In particular, the Sentinel-1 mission is a polar-orbiting satellite constellation for the acquisition of C-band Synthetic Aperture Radar (SAR) images. The spatial resolution will be of $5 \mathrm{~m}$, allowing a significant spatial step for most of the presented applications. As for the revisit time, the Sentinel-1 data acquisition concept is based on a systematic pre-defined acquisition approach. Such a choice will guarantee the chance of building consistent archives, and therefore the validity of the multi-temporal maps presented above.

The Sentinel 1 concept gives also the opportunity of acquiring wide swat products (Interferometric Wide (IW) and Extended Wide (EW) swath), so that the proposed approach could be scaled, in order to work on regional scales.

From the above presented technical and economic considerations, it emerges the opportunity of developing a capacity building for the effective use of SAR in humanitarian projects. The achievement of this opportunity requires only funds for the formation of local expertise.

\section{CONCLUSIONS}

In this paper we presented the potentialities and the future opportunities provided by the use of high resolution synthetic aperture radar data for humanitarian projects. A significant set of innovative products has been presented, with the support of the results obtained in the frame of a pilot project developed in the frame of the 2007 AO of the Italian sensor CosmoSkymed, a $3 \mathrm{~m}$ resolution satellite SAR. The project was developed with reference to the Yatenga region, located in the West African Sahel.

The presented products have been tailored on the Sahelian-Sudanese climate of the examined zone, which is characterized by a long dry season from October to April/May and intense rainfalls grouped between May/June and September with a peak of precipitations which usually occurs in August.

Level 2 products have been obtained by exploiting the specific climate, that leads to a dry scenario at the end of the dry season. Particular band combinations which allows the creation of false color images and indexes for enhancing some features of the scene such as the presence of vegetation, water or eroded areas have been shown.

In the near future, the applicative opportunities related with remote sensing application will be multiplied by the launch of the European Space Agency Sentinel mission, whose data will be provided via an open access policy. Such a scenario makes the use of high resolution SAR images still more attractive, because it removes the economic problems related with the image acquisition, that so far limited the diffusion of remote sensing applications in low-income countries.

\section{REFERENCES}

[1] C. Elachi and J. Van Zyl, Introduction to the Physics and Techniques of Remote Sensing, Wiley Ed., May 2006.

[2] F Ciervo, G Di Martino, A Iodice, Y Koussoube, MN Papa, D Riccio, G Ruello, I Zinno, "Use of high-resolution SAR data for the monitoring of water resources in Burkina Faso", SPIE Remote Sensing, 2011.

[3] Annor, F. O., van de Giesen, N., Liebe, J., van de Zaag, P., Tilmant, a., \& Odai, S. N. (2009). Delineation of small reservoirs using radar imagery in a semi-arid environment: A case study in the upper east region of Ghana. Physics and Chemistry of the Earth, Parts A/B/C, 34 (4-5), pp. 309-315.

[4] Amitrano D., Di Martino, G., Iodice, A.; Riccio, D.; Ruello, G.; Papa, M.N.; Ciervo, F.; Koussoube, Y., "High resolution SAR for monitoring of reservoirs sedimentation and soil erosion in semi arid regions", IEEE Geosci. and Remote Sensing Symposium, 2013.

[5] G. Di Martino, A. Iodice, A. Natale, D. Riccio, G. Ruello, I. Zinno, Y. Koussoube, M.N. Papa, F. Ciervo, "COSMOSkyMed AO projects - Use of high resolution SAR data for water resource management in semi arid regions", IEEE Geosci. and Remote Sensing Symposium, pp. 1212 - 1215, 2012.

[6] G. Di Martino, A. Iodice, D. Riccio, G. Ruello, Papa M. N., Koussoube Y., "Innovative Synthetic Aperture Radar Products for the Management of Land and Water", 2012 IEEE Global Humanitarian Technology Conference, p.117-121, 2012.

[7] Snoeij, P., E. Attema, M. Davidson, B. Duesmann, N. Floury, G. Levrini, B. Rommen, B. Rosich, "Sentinel-1 radar mission: Status and performance", IEEE Aerospace and Electronic Systems Magazine, vol. 25, pp. 32-39, 2010.

[8] B. Rosich, N. Miranda, P. Potin, C. Putignano, G. Sabella, D. Geudtner, "Sentinel-1 Ground Segment", IEEE Geosci. and Remote Sensing Symposium, pp. 1726-1729, 2012.

[9] R. Lal, "Soil erosion and conservation in West Africa", in World Soil Erosion and Conservation. Cambridge University Press, Cambridge, pp. 7-25, 1993.

[10] Wischmeier, W. H.; Smith, Predicting rainfall erosion losses a guide to conservation planning. Agricultural Handbook, vol. 537, U.S. Department of Agriculture, 1978. 